\title{
Poor prognostic factors of femoral shaft fractures in children treated by elastic intramedullary nailing
}

\author{
Alexandru Ulici ${ }^{1,2}$, Elena Odagiu ${ }^{1}$, Oana $\operatorname{Haram}^{1}$, Adelina Ionescu ${ }^{1}$, Gabriel Alin Sterian ${ }^{1,2}$, \\ Madalina Carp ${ }^{1, *}$, and Iulia Tevanov ${ }^{1}$ \\ ${ }^{1}$ Department of Pediatric Orthopedic Surgery, Emergency Hospital for Children “Grigore Alexandrescu”, 30-32 Iancu de Hunedoara \\ Blvd., 011733 Bucharest, Romania \\ 2 "Carol Davila" University of Medicine and Pharmacy, Bulevardul Eroii Sanitari 8, Bucureşti 050474, Romania
}

Received 30 April 2019, Accepted 11 August 2020, Published online 31 August 2020

\begin{abstract}
Introduction: Femoral shaft fractures in pediatric patients are treated by elastic intramedullary nailing using titanium or stainless-steel nails. The elastic stable intramedullary nailing behaves as an internal splint, promoting early mobilization. This type of treatment involves a minimally invasive approach, no damage to the growth plates, and no impairment of femoral head blood supply. Purpose: The aim of our study was to identify the negative predicting factors that might lead to an increased complication rate after elastic stable intramedullary nailing of femoral shaft fractures in children. Methods: We conducted a retrospective study on 137 patients with femoral shaft fractures treated by elastic stable intramedullary nailing. Patients' age ranged between 4 and 17 years. We used data from the medical records of the patients to evaluate postoperative complications. Plain radiographs were analyzed to determine the fracture type, fracture location, and postoperative complications such as delayed union, angular deformities, and limb length discrepancies. Multivariate analysis was conducted to identify predictors for poor outcomes. Results: Complications occurred in 29 patients $(21 \%)$ and consisted of delayed union, axial deformities, or lower limb length discrepancies. In the group of patients that suffered from complications, mechanism of injury, age, and weight were significant. They were older by an average of 5 years; half of them weighed more than $50 \mathrm{~kg}$ and over a half were involved in a road traffic accident. Conclusions: Elastic nailing is a successful tool to treat femoral shaft fractures. Three factors were demonstrated to influence the outcome. The mechanism of injury, age $>11$ years, and weight $>50 \mathrm{~kg}$ are the most important and are predictors for development of complications such as delayed union or deformity.
\end{abstract}

Key words: Femoral shaft fracture, Flexible intramedullary nail, Pediatric trauma, Pediatric orthopedics.

\section{Introduction}

Femoral shaft fractures represent $2 \%$ of all pediatric fractures and have a bimodal distribution based on patient's age [1]. Thus, in childhood, the femoral diaphysis is relatively weak and it could break during casual activities (childhood games), while throughout adolescence, $90 \%$ of the fractures are secondary to high energy trauma, such as motor vehicle accidents [2]. There are several factors that may influence the treatment of femoral shaft fractures including the age and weight of the child, associated lesions, type of fracture, surgeon's preferences, and socioeconomic status [1,2].

Closed reduction and spica cast immobilization is the best treatment for femoral shaft fractures in children aged less than 4 years [3]. In older patients, the use of different types of fixation is needed [4].
Fractures heal following the same stages in both children and adults; fractures in children are particular due to the osteogenic status of the pediatric bone and the healing process that is already ongoing when the bone fractures, while in adults the bone healing factors must be stimulated [5].

Elastic stable intramedullary nailing (ESIN) has become one of the main treatments for femoral shaft fractures in pediatric population. The elasticity of the nails allows the formation of external callus through micromovements at the fracture site [6]. This treatment does not have an impact on the growth plates and allows early mobilization [7]. Additionally, it has esthetic postoperative scars, facile removal, shortening of the inpatient period, good cost-effectiveness ratio, and less psychological impact on the patient $[7,8]$.

The insertion of the elastic nails in an antegrade or retrograde manner is influenced by the site of the fracture [9]. Fricka showed that the retrograde double-C shaped design of the nails provides greater resistance to deformation than antegrade $\mathrm{C}$ and $\mathrm{S}$ shaped model [9]. 
Lascombes et al. observed a low incidence of postoperative complications when the nails filled $80 \%$ of the medullary canal [10]. The French School emphasizes on the importance of nail contouring before insertion, so that the apex of the nail is at the fracture site [11]. The two nails must provide mutual support, preventing deformation by bending and ensuring rotational control [11].

Elastic nails do not provide rigid fixation at the fracture site $[10,12]$. Although this is beneficial in many children fractures, it has drawbacks in unstable fractures such as long spiral and comminuted fractures. These may shorten and angulate, mostly in overweight patients $(>45 \mathrm{~kg}$ ) and children who are older than 11 years of age $[12,13]$.

The complications following the ESIN treatment are relatively rare [14]. According to the literature, the most common complication is irritations at the protruded ends of the nail. This irritation may cause soft tissue and intraosseous infection. Other complications have been reported including nonunion, malunion with slight varus or valgus deformity, and limb length discrepancy. Recent studies show that an overgrowth of $1 \mathrm{~cm}$ occurs in $8.2 \%$ of preschool children [15]. Özdemir et al. analyzed limb length discrepancies and discovered an average difference of $1.8 \mathrm{~mm}$, with no clinical significance [16].

A few studies reported on poor prognostic factors that predict complications after ESIN [12, 13]. The aim of our study was to validate these studies and also to identify other potential poor predicting factors that might lead to an increased complication rate.

\section{Materials and method}

With the approval of the hospital's ethics committee (registrations number 8154), we conducted a retrospective study on 137 patients diagnosed with femoral shaft fracture who underwent close reduction and internal fixation using two elastic nails. The patients were admitted to our clinic between the 1st of January 2014 and the 31st of January 2018.

Our study included pediatric patients with femoral shaft fractures, aged between 4 and 17 years. We excluded pathological bone fractures (benign bone tumors, systemic diseases associated with bone fragility such as Osteogenesis Imperfecta etc.), open fractures, fractures that were treated by cast immobilization only, by open reduction and internal fixation, and other types of osteosynthesis such as internal fixation using plate and screws, external fixation, interlocking nail, etc.

We analyzed the patient's demographic data (age, gender, home environment, weight), side of injury (left/right), the location and type of fracture, and the occurrence of postoperative complications. Standard frontal and lateral view radiographs were evaluated to assess the stability of the fracture. Transverse and short oblique fractures are considered as stable fractures whereas long spiral and comminuted fractures are considered unstable. The site of fracture is grouped into proximal third, middle third, or distal third. The follow-up period was 12 months.

All the surgical procedures were performed by pediatric orthopedic surgeons under general anesthesia. After adequate closed reduction of the fracture, two titanium or stainless steel elastic nails were introduced. No endcaps were used.
The diameter of the nail varied between 2 and $4.5 \mathrm{~mm}$. The size of the nail was established by multiplying the narrowest diameter of the bone canal by 0.4 . The location of the fracture influenced the surgical approach. Thus, retrograde nailing was performed for middle or proximal shaft fractures, while antegrade approach was used for distal third fractures. In case of length unstable fractures, spica cast immobilization was used, for a period of time that ranged between 3 and 5 weeks. Nail removal was performed after a mean period of 10 months.

The callus formation was evaluated at 6 and 12 months on plain radiographs in standard views using Economedes' Method [17]; lower limb length discrepancies were determined on scanograms. Angulation deformities were measured on orthogonal radiographs by determining the angle between the anatomical axes of the bone fragments, distal and proximal to the fracture site, as seen in Figure 1.

Patients were divided into two groups: complicated and uncomplicated based on whether they did develop carefully selected complications or not. The selected complications included delayed union and deformities in any plane including leg length discrepancy. The values were compared to literature data of acceptable residual deformities following femoral fractures [18]. Patients with values outside the acceptable ranges are considered complicated.

The data was collected from patient's medical records and radiographs. Continuous data were recorded as mean, standard deviation (SD), and number of patients to indicate group size, with the treatment effect being reported as the mean difference (MD) with corresponding 95\% confidence interval (95\% CI). Dichotomous data were expressed as proportions, with the treatment effect reported as a risk ratio (RR) with 95\% CI. Statistical significance was set at $P<0.05$.

Several prognostic factors are considered including qualitative variables such as gender, home environment, fracture mechanism, location and fracture type, fracture stability, and quantitative variables such as age, weight. Based on their weight the patients were divided into two groups: less than $50 \mathrm{~kg}$ and over $50 \mathrm{~kg}$ because previous studies showed that children who are over $50 \mathrm{~kg}$ are at a higher risk of complication $[13,19]$. Univariate analysis was performed initially to understand the influence of individual factors on the outcome but generalized logistic regression models were also used to understand the interaction between the prognostic factors themselves. Statistical analysis was performed using the SPSS statistical software.

\section{Results}

The mean age for femoral fractures in our patients was 8 years (range: 4-17 years) with males involved as twice as females. The place of residence was predominantly urban $(64 \%)$. Forty percent of the fractures involved high energy trauma while others were caused by falls. The most common location of the fracture occurred in the middle third $(n=72$, $52 \%$ ). Seventy percent of the fractures were considered stable and $30 \%$ were unstable fractures. Seventy-two percent of the patient weighed less than $50 \mathrm{~kg}(n=99)$ whereas $28 \%$, weighed over $50 \mathrm{~kg}(n=38)$. 


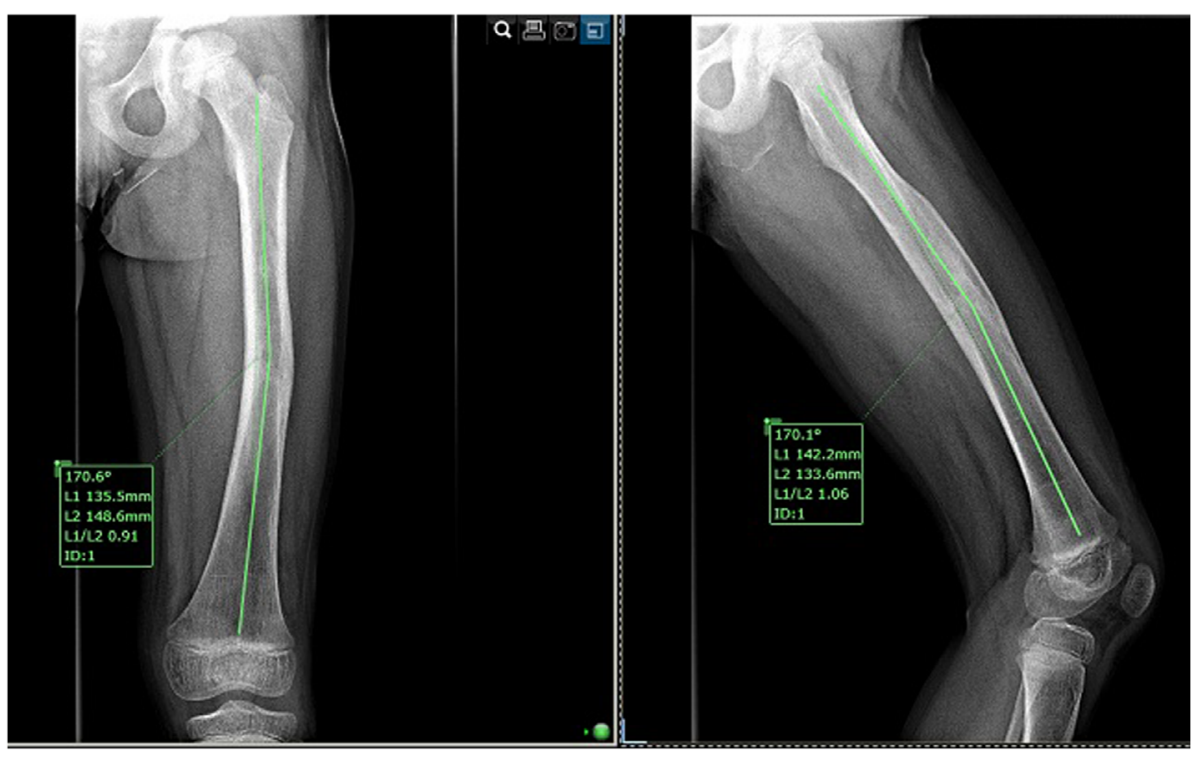

Figure 1. An 11-year-old patient with sagittal deformity $\left(9.4^{\circ}\right.$ procurvatum $)$ and coronal deformity $\left(11.2^{\circ}\right.$ varus $), 18$ months after elastic stable intramedullary nailing for a mid-shaft femoral fracture.

Table 1. Univariate analysis of the prognostic factors.

\begin{tabular}{lccc}
\hline Factors & Complicated & Uncomplicated & $\chi^{2}: P$-value \\
\hline Mechanism of injury (RTA/non RTA) & $16 / 13$ & $31 / 77$ & 0.008 \\
Stability (stable/unstable) & $11 / 18$ & $30 / 78$ & 0.29 \\
Approach (antegrade/retrograde) & $9 / 20$ & $22 / 86$ & 0.225 \\
Fracture site (proximal/middle/distal) & $7 / 14 / 8$ & $29 / 58 / 21$ & 0.452 \\
Medullary canal filling $(<80 \% />80 \%)$ & $25 / 4$ & $97 / 11$ & 0.582 \\
Weight $(<50 />50 \mathrm{~kg}$ ) & $10 / 19$ & $89 / 19$ & $<\mathbf{0 . 0 0 1}$ \\
Age (under 11/above 11) & $10 / 19$ & $89 / 19$ & $<\mathbf{0 . 0 0 1}$ \\
Gender (male/female) & $17 / 12$ & $76 / 32$ & 0.231 \\
\hline
\end{tabular}

*In bold the $P$-values under 0.05 .

Most of our patients $(n=108,79 \%)$ were successfully treated by closed reduction and internal fixation using elastic nails without any complication. This group of patients is categorized as the uncomplicated group. Selected complications (delayed union and deformities) occurred in 29 patients (21\%) and this comprises the complicated group.

Univariate analysis shows that site of fractures, fracture stability, and gender of the patient did not influence complication rates. The surgical approach whether antegrade or retrograde or the size of the nail used in relation to the size of the femoral shaft medullary canal did not cause more complications as well. However, three factors were associated with significantly higher complication rates. Children who were over 11 years old and those who weighed more than $50 \mathrm{~kg}$ had significantly more complications rates $\left(\chi^{2}: P\right.$-value $\left.<0.001\right)$. Children whose fractures were caused by road traffic accidents were at a higher risk of complications $\left(\chi^{2}: P\right.$-value $\left.=0.008\right)($ Table 1$)$.

In our patients, there was a strong association between age and weight with $97 \%(n=96)$ of patients who are under the age of 11 years weighing less than $50 \mathrm{~kg}$ whereas $92 \%(n=35)$ of the children who were over 11 years weighed over $50 \mathrm{~kg}$. This was statistically significant $\left(\chi^{2}: P\right.$-value $\left.<0.001\right)$. Furthermore, $64 \%$ of children who are younger than 11 years old were involved in road traffic accident in comparison to $36 \%$. This did not reach statistical significance $\left(\chi^{2}: P\right.$-value $\left.=0.112\right)$. Given the interplay between these three important prognostic factors, a multivariate analysis demonstrated that the only significant factor for complication was the mechanism of injury $(P$-value $=0.029)($ Table 2$)$.

\section{Discussions}

The elastic stable intramedullary nailing behaves as an internal splint, promoting an early mobilization of the patient. This type of treatment involves a minimally invasive approach, no damage to the growth plates and no impairment of femoral head blood supply [20]. Over the past 10 years, intramedullary nailing using steel or titanium elastic nails has been promoted in a double C-shaped fashion [11]. To determine the negative prognostic factors and the complication rates of femoral shaft fractures in children treated by ESIN, we performed this retrospective study.

In our study, the univariate analysis showed a statistically significant relationship between age, weight, mechanism of injury, and complication rates. The mean age in the group of patients that suffered complications was 11 years and 2 months, 
Table 2. Multivariate analysis of the prognostic factors for femoral shaft fracture.

\begin{tabular}{|c|c|c|c|c|c|c|c|}
\hline \multirow[t]{2}{*}{ Factors } & \multirow[t]{2}{*}{$B$} & \multirow[t]{2}{*}{$S E$} & \multirow[t]{2}{*}{ Wald } & \multirow[t]{2}{*}{$P$-value } & \multirow[t]{2}{*}{$\operatorname{Exp}(B)$} & \multicolumn{2}{|c|}{$95 \%$ CI for $\operatorname{Exp}(B)$} \\
\hline & & & & & & Lower & Upper \\
\hline Mechanism of injury & 1.139 & 0.521 & 4.778 & 0.029 & 3.124 & 1.125 & 8.676 \\
\hline Stability & -0.549 & 0.538 & 1.042 & 0.307 & 0.577 & 0.201 & 1.658 \\
\hline Approach & -1.347 & 0.970 & 1.928 & 0.165 & 0.260 & 0.039 & 1.741 \\
\hline Fracture site & 0.414 & 0.600 & 0.476 & 0.490 & 1.513 & 0.467 & 4.904 \\
\hline Medullary canal filling & 1.831 & 1.150 & 2.534 & 0.111 & 6.238 & 0.655 & 59.408 \\
\hline Weight & 0.885 & 0.759 & 1.361 & 0.243 & 2.424 & 0.548 & 10.726 \\
\hline Age & 0.689 & 1.127 & 0.374 & 0.541 & 1.991 & 0.219 & 18.121 \\
\hline Gender & -0.626 & 0.535 & 1.369 & 0.242 & 0.534 & 0.187 & 1.527 \\
\hline
\end{tabular}

while, the mean age in the group of patients without complications was 6 years. Out of the 29 patients who experienced postoperative complications, 19 were weighting more than $50 \mathrm{~kg}(65.5 \%)$. Fifty-five percent of patients who developed complications had road traffic accident as a cause of their fracture in comparison to $29 \%$ only in the uncomplicated group. Moroz and colleagues reported similar findings regarding the weight and age [19]. In their study, they found out that "a poor outcome was five times more likely in children who weighed more than $49 \mathrm{~kg}$ " [19]. Sagan et al. determined in their research on 70 fractures treated by ESIN, that a mean weight of $46.5 \mathrm{~kg}$ was an important predictive factor of an anterior bow deformity greater than $15^{\circ}[21]$.

The new interesting finding of our study is that the multivariate analysis demonstrated that the age and weight were dependent on the mechanism of injury which was more significant than both. As expected, weight and age are correlated with each other. The interplay between these two factors was demonstrated in our study with most patients who are over 11 years weighed more than $50 \mathrm{~kg}$. We anticipated a similar interplay between age and the frequency of road traffic accident with older children being more prone. Although, $64 \%$ of children who are younger than 11 years were involved in road traffic accident in comparison to $36 \%$, this did not reach statistical significance. This complex relationship among these important prognostic factors is further explored with multivariate analysis to determine the importance of each to predict outcome. We used a stepwise method for variable selection in logistic regression analysis and we found the mechanism of injury is an important factor, which is clinically plausible.

In the analyzed group, complications such as delay in consolidation, deformation in coronal and sagittal planes, or shortening of the affected limb occurred more often in the distal third femoral shaft fractures, 8 of $21(27.6 \%)$ distal third compared to 14 of $58(19.4 \%)$ in the middle third, but this finding was not statistically significant $(P=0.454)$. Moroz's study also showed a statistically nonsignificant result; poor outcome was determined in a higher percentage in fractures of the distal third, 6 of $33(18 \%)$, compared to 14 of $164(9 \%)$ in the middle third [19].

Regarding length stability, the fractures in our study were divided into two groups: length stable (transverse and short oblique) and unstable fractures (long oblique and comminuted). Some studies found that length unstable fractures can have a negative outcome because of the associated postoperative complications such as loss of reduction and malunion [22]. In his study on 39 patients, Sink concluded that "the complications that required unplanned surgery for either prominent nails or loss of reduction occurred more commonly in unstable nontransverse fracture patterns" [23]. According to our research, a higher stability of the fracture site does not correlate with lower rates of postoperative complications $(P=0.293)$, compared to unstable fractures. Based on our finding, unstable femur fractures treated by ESIN and spica cast immobilization are not at an increased risk of more complications such as angular deformities, delayed union, limb length discrepancies, or higher premature nail removal rates.

We evaluated the callus formation according to Economedes' method, where at least three cortical edges are visibly united on the radiographs [17]. The callus formation was checked at 6 months and 12 months on plain radiographs in standard views (frontal and lateral). The results were compared to medical literature. A delayed union was considered when less than three cortical edges were visibly united on the radiograph at the 6-month follow-up; a delayed union that persisted at the 12-month follow-up was defined as a pseudarthrosis or nonunion $[19,21]$. In our study, one of the postoperative complications consisted of delayed union $(n=13$, $9.48 \%$ ). Seven patients that suffered from delayed union had other associated angular deformities at the 6-month follow-up. These deformities persisted at the 12-month follow-up, but none of the fractures emerged toward a nonunion.

According to the literature, the most common early complications are soft tissue lesions because of nail prominence and nail migration [20]. Knee pain, bursitis, and limitation of the knee flexion could not be evaluated in our research, because there were no recorded data for these symptoms. Several researches encountered more severe complications because of nail prominence such as skin ulcerations and deep wound infections $[24,25]$. To prevent these types of complications, endcaps can be used in order to protect the skin from the sharp cutting end of the nail and allow fracture healing without further nail migration and bone telescoping [26]. In our study, there were no cases of skin perforation because of nail prominence that needed a revision procedure or premature nail removal. Endcaps were not used for the patients included in our study.

\section{Conclusion}

Elastic nailing is a successful tool to treat femoral shaft fractures. Majority they do well. Three factors were demonstrated 
to influence the outcome. The mechanism of injury, age $>$ 11 years, and weight $>50 \mathrm{~kg}$ with the mechanism of injury being the most important and the only independent predictor for development of complications such as delayed union or deformity.

\section{Conflict of interest}

The authors certify that they have no financial conflict of interest (e.g., consultancies, stock ownership, equity interest, patent/licensing arrangements, etc.) in connection with this article.

This research received no specific grant from any funding agency in the public, commercial or not-for-profit sectors.

This article does not contain any studies with human participants or animals performed by any of the authors. This article received the approval of our Review Ethics committee, registered with the number: 8154 . Informed consent was obtained from all individual participants included in the study.

Acknowledgements. We gratefully thank Dr. Sattar Alshryda (Central Manchester University Hospitals NHS Foundation Trust) for his assistance with methodology and statistical research and for his comments that greatly improved our manuscript.

\section{References}

1. Rewers A, Hedegaard H, Lezotte D, et al. (2005) Childhood femur fractures, associated injuries, and sociodemographic risk factors: a population-based study. Pediatrics 115(5), e543-e552.

2. Hoffmann CR, Traldi EF, Posser A (2012) Epidemiological study of children diaphyseal femoral fractures. Rev Bras Ortop Engl Ed 47(2), 186-190.

3. Carey TP, Galpin RD (1996) Flexible intramedullary nail fixation of pediatric femoral fractures. Clin Orthop 332, 110-118.

4. Letts M, Jarvis J, Lawton L, Davidson D (2002) Complications of rigid intramedullary rodding of femoral shaft fractures in children. J Trauma 52, 504-516.

5. Tevanov I, Enescu DM, Carp M, Dusca A, Ladaru A, Ulici A (2018) Negative pressure wound therapy in reconstructing extensive leg and foot soft tissue loss in a child: a case study. J Wound Care 27(Sup6), S14-S19.

6. Noordeen MH, Lavy CB, Shergill NS, Tuite JD, Jackson AM (1995) Cyclical micromovement and fracture healing. J Bone Joint Surg Br 77(4), 645-648.

7. Tochie JN, Guifo ML, Yamben MAN, Moulion R, Farikou I (2017) A prospective cohort study of the therapeutic patterns, challenges and outcomes of paediatric femoral fractures in a Cameroonian Tertiary Center. Open Orthop J 11, 29-36.

8. Saikia K, Bhuyan S, Bhattacharya T, Saikia S (2007) Titanium elastic nailing in femoral diaphyseal fractures of children in 16 years of age. Indian J Orthop 41(4), 381-385.

9. Fricka KB, Mahar AT, Lee SS, Newton PO (2004) Biomechanical analysis of antegrade and retrograde flexible intramedullary nail fixation of pediatric femoral fractures using a synthetic bone model. J Pediatr Orthop 167-171.

10. Lascombes P, Huber H, Fay R (2013) Flexible intramedullary nailing in children: nail to medullary canal diameters optimal ratio. J Pediatr Orthop 33, 403-408.

11. Lascombes P, Haumont T, Journeau P (2006) Use and abuse of flexible intramedullary nailing in children and adolescents. J Pediatr Orthop 26, 827-834.

12. Deakin DE, Winter H, Jain P, Bache CE (2010) Malunion following flexible intramedullary nails for tibial and femoral fractures in adolescents. J Child Orthop 4(6), 571-577.

13. Li Y, Stabile KJ, Shilt JS (2008) Biomechanical analysis of titanium elastic nail fixation in a pediatric femur fracture model. Journal of Pediatric Orthopaedics 28(8), 874-878.

14. Luhmann SJ, Schootman M, Schoenecker PL, Dobbs MB, Gordon JE (2003) Complications of titanium elastic nails for pediatric femoral shaft fractures. J Pediatr Orthop 23(4), 443-447.

15. Bopst L, Reinberg O, Lutz N (2007) Femur fracture in preschool children. J Pediatr Orthop 27(3), 299-303.

16. Özdemir HM, Yensel U, Senaran H, Mutlu M, Kutlu A (2003) Immediate percutaneous intramedullary fixation and functional bracing for the treatment of pediatric femoral shaft fracture. J Pediatr Orthop 23(4), 453-457.

17. Economedes DM, Abzug JM, Paryayi E, Hermann MJ (2014) Outcomes using titanium elastic nails for open and close pediatric tibia fractures. Orthopedics 37(7), e619-e624.

18. Flynn JM, Skaggs DL (2015) Chapter 27: Femoral shaft fractures. In Rockwood and Wilkins' fractures in children, 8th edn. Flynn JM, Skaggs DL, Waters PM, Editors. Wolters Kluwer Healt: Philadelphia, PA.

19. Moroz LA, Launay F, Kocher MS, Newton PO, Frick SL, Sponseller PD, Flynn JM (2006) Titanium elastic nailing of fractures of the femur in children. Predictors of complications and poor outcome. J Bone Joint Surg Br 88, 1361-1366.

20. Mahar A, Sink E, Faro F, Oka R, Newton PO (2007) Differences in biomechanical stability of femur fracture fixation when using titanium nails of increasing diameter. J Child Orthop 1(3), 211-215.

21. Sagan ML, Datta JC, Olney BW, Lansford TJ, McIff TE (2010) Residual deformity after treatment of pediatric femur fractures with flexible titanium nails. J Pediatr Orthop 30(7), 638-643.

22. Parikh SN, Jain VV, Denning J, et al. (2012) Complications of elastic stable intramedullary nailing in pediatric fracture management: AAOS exhibit selection. J Bone Joint Surg Am 94(24), e184.

23. Sink EL, Gralla J, Repine M (2005) Complications of Pediatric Femur Fractures Treated With Titanium Elastic Nails. J Pediatr Orthop 25(5), 577-580.

24. Ligier J, Metaizeau J, Prevot J, Lascombes P (1988) Elastic stable intramedullary nailing of femoral shaft fractures in children. J Bone Joint Surg Br. 70-B(1), 74-77.

25. Flynn JM, Hresko T, Reynolds RAK, Blasier RD, Davidson R, Kasser J (2001) Titanium elastic nails for pediatric femur fractures: a multicenter study of early results with analysis of complications. J Pediatr Orthop 21(1), 4-8.

26. Nectoux E, Giacomelli MC, Karger C, Gicquel P, Clavert JM (2008) Use of end caps in elastic stable intramedullary nailing of femoral and tibial unstable fractures in children: preliminary results in 11 fractures. J Child Orthop 4, 309-314.

Cite this article as: Ulici A, Odagiu E, Haram O, Ionescu A, Sterian GA, Carp M \& Tevanov I (2020) Poor prognostic factors of femoral shaft fractures in children treated by elastic intramedullary nailing. SICOT-J 6, 34 